\title{
Best bounds for dispersion of ratio block sequences for certain subsets of integers
}

\author{
József Bukor, Peter Csiba
}

Department of Mathematics and Informatics

J. Selye University, Komárno, Slovakia

bukorj@ujs.sk

csibap@ujs.sk

Submitted January 25, 2018 - Accepted May 28, 2018

\begin{abstract}
In this paper, we study the behavior of dispersion of special types of sequences which block sequence is dense.
\end{abstract}

Keywords: block sequence, dispersion, $(R)$-density

$M S C: 11 \mathrm{~B} 05$

\section{Introduction}

Denote by $\mathbb{N}$ and $\mathbb{R}^{+}$the set of all positive integers and positive real numbers, respectively. Let $X=\left\{x_{1}<x_{2}<x_{3}<\cdots\right\}$ be an infinite subset of $\mathbb{N}$. Denote by $R(X)=\left\{\frac{x_{i}}{x_{j}}: i, j \in \mathbb{N}\right\}$ the ratio set of $X$, and say that a set $X$ is $(R)$-dense if $R(X)$ is (topologically) dense in the set $\mathbb{R}^{+}$. The concept of $(R)$-density was introduced by T. Šalát [7].

The following sequence of finite sequences derived from $X$

$$
\frac{x_{1}}{x_{1}}, \frac{x_{1}}{x_{2}}, \frac{x_{2}}{x_{2}}, \frac{x_{1}}{x_{3}}, \frac{x_{2}}{x_{3}}, \frac{x_{3}}{x_{3}}, \ldots, \frac{x_{1}}{x_{n}}, \frac{x_{2}}{x_{n}}, \ldots, \frac{x_{n}}{x_{n}}, \ldots
$$

is called the block sequence of the sequence $X$.

It is formed by the blocks $X_{1}, X_{2}, \ldots, X_{n}, \ldots$ where

$$
X_{n}=\left(\frac{x_{1}}{x_{n}}, \frac{x_{2}}{x_{n}}, \ldots, \frac{x_{n}}{x_{n}}\right), \quad n=1,2, \ldots
$$


is called the $n$-th block. This kind of block sequences was introduced by O. Strauch and J. T. Tóth [9].

For each $n \in \mathbb{N}$ consider the step distribution function

$$
F\left(X_{n}, x\right)=\frac{\#\left\{i \leq n ; \frac{x_{i}}{x_{n}}<x\right\}}{n},
$$

and define the set of distribution functions of the ratio block sequence

$$
G\left(X_{n}\right)=\left\{\lim _{k \rightarrow \infty} F\left(X_{n_{k}}, x\right)\right\} .
$$

The set of distribution functions of ratio block sequences was studied in $[1,2,5,6$, $8,12]$.

For every $n \in \mathbb{N}$ let

$$
D\left(X_{n}\right)=\max \left\{\frac{x_{1}}{x_{n}}, \frac{x_{2}-x_{1}}{x_{n}}, \ldots, \frac{x_{i+1}-x_{i}}{x_{n}}, \ldots, \frac{x_{n}-x_{n-1}}{x_{n}}\right\},
$$

the maximum distance between two consecutive terms in the $n$-th block. We will consider the quantity

$$
\underline{D}(X)=\liminf _{n \rightarrow \infty} D\left(X_{n}\right),
$$

(see [10]) called the dispersion of the block sequence (1.1) derived from $X$. Relations between asymptotic density and dispersion were studied in [11].

The aim of this paper is to study the behavior of dispersion of the block sequence derived from $X$ under the assumption that $X=\bigcup_{n=1}^{\infty}\left(c_{n}, d_{n}\right\rangle \cap \mathbb{N}$ is $(R)$-dense and the limit $\lim _{n \rightarrow \infty} \frac{d_{n}}{c_{n}}=s$ exists. In this case

$$
\underline{D}(X) \leq \begin{cases}\frac{1}{s+1}, & \text { if } s \in\left\langle 1, \frac{1+\sqrt{5}}{2}\right) \\ \frac{1}{s^{2}}, & \text { if } s \in\left\langle\frac{1+\sqrt{5}}{2}, 2\right) \\ \frac{s-1}{s^{2}}, & \text { if } s \in\langle 2, \infty)\end{cases}
$$

(see [10, Theorem 10]). This upper bound for $\underline{D}(X)$ is the best possible if $s \geq 2$ (see [4]) and in the case $\frac{1+\sqrt{5}}{2} \leq s \leq 2$ (see [3]). We prove that the above upper bound for $\underline{D}(X)$ is also optimal in the remainding case $1 \leq s<\frac{1+\sqrt{5}}{2}$, i.e. $\underline{D}(X)$ can be any number in the interval $\left\langle 0, \frac{1}{s+1}\right\rangle$.

\section{Results}

First, we show that there is a connection between the dispersion and the distribution functions of a ratio block sequence.

Theorem 2.1. Let $X \subset \mathbb{N}$, and assume that the dispersion $\underline{D}(X)$ of the related block sequence is positive. Let $g \in G\left(X_{n}\right)$. Then $g$ is constant on an interval of length $\underline{D}(X)$. 
Proof. Let $\varepsilon<\underline{D}(X)$ be an arbitrary positive real number. By the definition of dispersion it follows that for sufficiently large $n$ the step distribution function $F\left(X_{n}, x\right)$ is constant on some interval $\left(\frac{x_{i}}{x_{n}}, \frac{x_{i+1}}{x_{n}}\right\rangle$ of length $\underline{D}(X)-\varepsilon$. A simple compactness argument yields that there exist

- real numbers $\gamma, \delta \in\langle 0,1\rangle$ such that $\delta-\gamma \geq \underline{D}(X)-\varepsilon$,

- an increasing sequence $\left(n_{k}\right)$ and a sequence $\left(m_{k}\right)$ of positive integers such that $m_{k}<n_{k}$

$$
\lim _{k \rightarrow \infty} \frac{x_{m_{k}}}{x_{n_{k}}}=\gamma, \quad \lim _{k \rightarrow \infty} \frac{x_{m_{k}+1}}{x_{n_{k}}}=\delta \quad \text { and } \quad \lim _{k \rightarrow \infty} F\left(X_{n_{k}}, x\right)=g(x) \text { a.e. on }\langle 0,1\rangle \text {. }
$$

Hence $g$ is constant on the interval $(\gamma, \delta)$ of length $\underline{D}(X)-\varepsilon$. Since $\varepsilon$ can be chosen arbitrary small, and the assertion of the theorem follows.

The next lemma is useful for the determination of the value of the dispersion $\underline{D}(X)$ (see [10, Theorem 1]).

Lemma 2.2. Let

$$
X=\bigcup_{n=1}^{\infty}\left(c_{n}, d_{n}\right\rangle \cap \mathbb{N},
$$

and for $n \in \mathbb{N}$ let $c_{n}<d_{n}<c_{n+1}$ be positive integers. Then

$$
\underline{D}(X)=\liminf _{n \rightarrow \infty} \frac{\max \left\{c_{i+1}-d_{i}: i=1, \ldots, n\right\}}{d_{n+1}} .
$$

For the proof of $(R)$-density we shall use the following lemma.

Lemma 2.3. Denote by $\left(p_{n}\right),\left(q_{n}\right),\left(u_{n}\right),\left(v_{n}\right),\left(w_{n}\right)$ and $\left(z_{n}\right)$ be strictly increasing sequences of positive integers satisfying

$$
p_{n}<q_{n}<u_{n}<v_{n} \quad \text { and } \quad w_{n}<z_{n}, \quad(n=1,2,3, \ldots) .
$$

Further, let

$$
\left(\frac{q_{n}}{p_{n}}\right),\left(\frac{u_{n}}{p_{n}}\right),\left(\frac{v_{n}}{u_{n}}\right),\left(\frac{w_{n}}{u_{n}}\right) \text { and }\left(\frac{z_{n}}{w_{n}}\right)
$$

converge to real numbers greater than 1 , moreover

$$
\lim _{n \rightarrow \infty} \frac{z_{n}}{w_{n}} \geq \lim _{n \rightarrow \infty} \frac{u_{n}}{q_{n}}
$$

Then the ratio set of

$$
\bigcup_{n}\left(\left(p_{n}, q_{n}\right\rangle \cup\left(u_{n}, v_{n}\right\rangle \cup\left(w_{n}, z_{n}\right\rangle\right) \cap \mathbb{N}
$$

is dense on the interval

$$
\left\langle\lim _{n \rightarrow \infty} \frac{w_{n}}{v_{n}}, \lim _{n \rightarrow \infty} \frac{z_{n}}{p_{n}}\right\rangle .
$$


The proof is elementary and we leave it to the reader. Let us suppose that $k \in \mathbb{N}$ is a constant. Note that the assertion of the lemma remains still true if one removes $k$ elements from the sets $\left(u_{n}, v_{n}\right\rangle \cap \mathbb{N}$ for all sufficiently large $n$.

The main result of this paper is the following.

Theorem 2.4. Let $s \in\left(1, \frac{1+\sqrt{5}}{2}\right)$ be an arbitrary real number. Then for any $\alpha \in\left\langle 0, \frac{1}{s+1}\right\rangle$ there is an $(R)$-dense set

$$
X=\bigcup_{n=1}^{\infty}\left(c_{n}, d_{n}\right\rangle \cap \mathbb{N},
$$

where $c_{n}<d_{n}<c_{n+1}$ are positive integers for that $\lim _{n \rightarrow \infty} \frac{d_{n}}{c_{n}}=s$ and $\underline{D}(X)=\alpha$.

Proof. It was shown in [4, Theorem 2] that the dispersion $\underline{D}(X)$ can take any number in the interval $\left\langle 0, \frac{s-1}{s^{2}}\right\rangle$. In what follows we suppose $\frac{s-1}{s^{2}}<\alpha \leq \frac{1}{s+1}$. Let us consider the function $f(x)=\frac{x-1}{s x}$. Clearly, this function is continuous and increasing on the interval $\langle 1, \infty)$. Moreover

$$
f(s)=\frac{s-1}{s^{2}} \quad \text { and } \quad f(s+1)=\frac{1}{s+1} .
$$

Thus, there exists a real number $t \in(s, s+1\rangle$ with the property

$$
\frac{t-1}{s t}=\alpha \text {. }
$$

Write $\frac{1}{\alpha}$ in the form $s^{k+\delta}$, where $k$ is an integer and $0 \leq \delta<1$. The lower bound $k \geq 2$ follows from the facts that $s+1 \leq \frac{1}{\alpha}$ and $s+1 \geq s^{2}$ whenever $1<s \leq \frac{1+\sqrt{5}}{2}$.

Define the set $X \subset \mathbb{N}$ by

$$
X=\bigcup_{n=1}^{\infty}\left(A_{n} \cup B_{n}\right) \cap \mathbb{N},
$$

where

$$
A_{n}=\bigcup_{i=1}^{k}\left(a_{n, i}, b_{n, i}\right\rangle \quad \text { and } \quad B_{n}=\bigcup_{j=1}^{n}\left(c_{n, j}, d_{n, j}\right\rangle
$$

Put $a_{1,1}=1$ and

$$
\begin{aligned}
& b_{n, i}=\left[s . a_{n, i}\right] \text { for } n \in \mathbb{N} \text { and } i=1,2, \ldots, k, \\
& a_{n, i}= \begin{cases}d_{n-1, n-1} ! & \text { for } n \geq 2, i=1 \\
{\left[s^{\delta} . b_{n, 1}\right]+1} & \text { for } n \in \mathbb{N}, i=2 \\
b_{n, i-1}+1 & \text { for } n \in \mathbb{N}, i=3, \ldots, k,\end{cases} \\
& c_{n, j}= \begin{cases}{\left[t . b_{n, k}\right]+1} & \text { for } n \in \mathbb{N}, j=1 \\
{\left[t . d_{n, j-1}\right]+1} & \text { for } n \in \mathbb{N}, j=2, \ldots, n,\end{cases}
\end{aligned}
$$




$$
d_{n, j}=\left[s . c_{n, j}\right] \quad \text { for } n \in \mathbb{N} \text { and } j=1,2, \ldots, n \text {. }
$$

First we prove that $\underline{D}(X)=\alpha$. For sufficiently large $n$, by the definition of the set $X$ we have the inequalities

$$
\begin{aligned}
a_{n+1,1}-d_{n, n} & >c_{n, n}-d_{n, n-1}>c_{n, n-1}-d_{n, n-2}>\cdots \\
& >c_{n, 3}-d_{n, 2}>c_{n, 2}-d_{n, 1}>c_{n, 1}-b_{n, k}
\end{aligned}
$$

further

$$
a_{n, 1}-d_{n-1, n-1}<c_{n, 1}-b_{n, k}
$$

and

$$
a_{n, 2}-b_{n, 1}<a_{n, 1}-d_{n-1, n-1} .
$$

Observe that inequality $(2.3)$ holds if $\frac{1}{\alpha}(t-1)>1$. In virtue of $(2.1)$ this is equivalent with $s t>1$, which evidently holds. As

$$
s^{1+\delta}-s-1<s^{2}-s-1
$$

and $s^{2}-s-1$ is negative for $s \in\left(1, \frac{1+\sqrt{5}}{2}\right)$, inequality (2.4) follows.

Now we use Lemma 1. From the inequalities $(2.2-2.4)$ one can see that it is sufficient to study the quotients
a) $\frac{a_{n, 1}-d_{n-1, n-1}}{b_{n, k}}$
b) $\frac{c_{n, 1}-b_{n, k}}{d_{n, 1}}$
c) $\frac{c_{n, k}-d_{n, k-1}}{d_{n, k}}$

In case a) we see

$$
\liminf _{n \rightarrow \infty} \frac{a_{n, 1}-d_{n-1, n-1}}{b_{n, k}}=\liminf _{n \rightarrow \infty} \frac{a_{n, 1}}{\frac{1}{\alpha} a_{n, 1}}=\alpha,
$$

in case b)

$$
\liminf _{n \rightarrow \infty} \frac{c_{n, 1}-b_{n, k}}{d_{n, 1}}=\liminf _{n \rightarrow \infty} \frac{t b_{n, k}-b_{n, k}}{s t b_{n, k}}=\frac{t-1}{s t}=\alpha,
$$

and the remaining case c) is analogous to case b).

It remains to prove that the set $X$ is $(R)$-dence. Using Lemma 2 we show that the ratio set of the set $X$ is dense on the intervals

$$
\begin{aligned}
& \left\langle 1, \frac{1}{\alpha}\right\rangle\left(\text { for } p_{n}=a_{n, 1}, q_{n}=b_{n, 1}, u_{n}=w_{n}=a_{n, 2}, v_{n}=z_{n}=b_{n, k}\right), \\
& \left\langle t^{i} s^{i-1}, \frac{1}{\alpha} s^{i} t^{i}\right\rangle\left(\text { for } p_{n}=a_{n, 1}, q_{n}=b_{n, 1}, u_{n}=a_{n, 2}, v_{n}=b_{n, k}, w_{n}=c_{n, i}, z_{n}=d_{n, i}\right) .
\end{aligned}
$$

Hence, by $\frac{1}{\alpha} \geq s+1$ and $t<s+1$ we have

$$
\left\langle 1, \frac{1}{\alpha}\right\rangle \cup \bigcup_{i=1}^{\infty}\left\langle t^{i} s^{i-1}, \frac{1}{\alpha} s^{i} t^{i}\right\rangle=\langle 1, \infty),
$$

and therefore the $(R)$-density of the set $X$ follows. 


\section{References}

[1] Baláž, V., Mišík, L., Strrauch, O., Tóth, J. T., Distribution functions of ratio sequences, III, Publ. Math. Debrecen, 82 (2013), 511-529.

https://doi.org/10.5486/pmd.2013.4770

[2] Baláž, V., Mišík, L., Strrauch, O., Tóth, J. T., Distribution functions of ratio sequences, IV, Period. Math. Hung., 66 (2013), 1-22.

https://doi.org/10.1007/s10998-013-4116-4

[3] Filip, F., Liptai, K., Mátyás, F., Tóth, J. T., On the best estimations for dispersions of special ratio block sequences, Ann. Math. Inf., 37 (2010), 85-93.

[4] Filip, F., Tóth, J. T., On estimations of dispersions of certain dense block sequences, Tatra Mt. Math. Publ., 31 (2005), 65-74.

[5] Grekos, G., Strauch, O., Distribution functions of ratio sequences, II, Unif. Distrib. Theory 2 (2007), 53-77.

[6] KrČmarský, D., Mišík, L., Václavíková, Z., On small set of distribution functions of ratio block sequences Unif. Distrib. Theory 11 (2016), 165-174.

https://doi.org/10.1515/udt-2016-0009

[7] ŠAlát, T., On ratio sets of sets of natural numbers, Acta Arith., 15 (1969), 273-278. https://doi.org/10.4064/aa-15-3-273-278

[8] Strauch, O., Distribution functions of ratio sequences. An expository paper, Tatra Mt. Math. Publ., 64 (2015), 133-185. https://doi.org/10.1515/tmmp-2015-0047

[9] Strauch, O., Tóth, J. T., Distribution functions of ratio sequences, Publ. Math. Debrecen, 58 (2001), 751-778.

[10] Tо́тн, J. T., Mišík, L., Filip, F., On some properties of dispersion of block sequences of positive integers, Math. Slovaca, 54 (2004), 453-464.

[11] Tóth, J. T., Mišík, L., Filip, F., Dispersion of ratio block sequences and asymptotic density, Acta Arith., 131 (2008), 183-191.

https://doi.org/10.4064/aa131-2-5

[12] Tо́тн, J. T., Mišík, L., Filip, F., On ratio block sequences with extreme distribution function, Math. Slovaca, 59 (2009), 275-282.

https://doi.org/10.2478/s12175-009-0123-6 